\section{Evaluation of extended spectrum beta lactamase enzymes prevalence in clinical isolates of Escherichia coli}

\author{
Ronak Bakhtiari,' Jalil Fallah Mehrabadi, \\ Hedroosha Molla Agamirzaei, ${ }^{3}$ \\ Ailar Sabbaghi, ${ }^{3}$ Mohammad Mehdi \\ Soltan Dallal, \\ 1Dev. Microbiology, Department of \\ Pathobiology, School of Public Health, \\ Tehran University of Medical Science; \\ 2MARS Bioinformatics Institute, Tehran; \\ ${ }^{3}$ Faculty of Basic and Medical Sciences, \\ Department of Microbiology, Islamic \\ Azad University, Zanjan Branch, Zanjan; \\ ${ }^{4}$ Antibiotic Resistant Research Center, \\ Tehran University of Medical Sciences, \\ Tehran, Iran
}

\section{Abstract}

Resistance to $\beta$-lactam antibiotics by gramnegative bacteria, especially Escherichia coli (E. coli), is a major public health issue worldwide. The predominant resistance mechanism in gram negative bacteria particularly $E$. coli is via the production of extended spectrum beta lactamase (ESBLs) enzymes. In recent years, the prevalence of $\beta$-lactamase producing organisms is increased and identification of these isolates by using disk diffusion method and no-one else is not satisfactory. So, this investigation focused on evaluating the prevalence of ESBL enzymes by disk diffusion method and confirmatory test (Combined Disk). Five hundred clinical samples were collected and $200 \mathrm{E}$. coli isolates were detected by standard biochemical tests. To performing initial screening of ESBLs was used from Disk diffusion method on $E$. coli isolates. A confirmation test (Combined Disk method) was performed on isolates of resistant to cephalosporin's indicators. Up to 70\% isolates exhibited the Multi Drug Resistance phenotype. In Disk diffusion method, 128(64\%) $E$. coli isolates which resistant to ceftazidime and cefotaxime while in Combined Disk, among 128 screened isolates, 115 (89.8\%) isolates were detected as ESBLs producers. This survey indicate beta lactamase enzymes are playing a significant role in antibiotic resistance and correct detection of them in phenotypic test by using disk diffusion and combined Disk is essential for accurate recognition of ESBLs.

\section{Introduction}

Different strategies are used to protect bacteria from destructive effects of antibiotics. ${ }^{1}$ One of the most important resistant mechanisms by bacteria against antibiotics is production of enzymes that hydrolyze the beta lactam ring of antibiotics. The action of these antibiotics is via the inhibition of cell wall synthesis and bacterial cell division. ${ }^{2}$ Extended spectrum beta lactamase (ESBLs) are specific types of beta lactamases with particular mutation, making them capable of destroying expanded cephalosporin (cefotaxime, ceftazidime) and azetreonam, but don't have activity against carbapenems (imipenem), cephamycins (cephoxitin) and beta lactam inhibitor (clavulanic acid, sulbactam). ${ }^{3}$ On the other hand, development of novel antibiotics and promotion of their use in the treatment of bacterial infectious diseases led to the appearance of newer enzyme classes in beta lactamase super family particularly AmpC. ${ }^{4}$ ESBLs enzymes were discovered in early 1980 and these are classified based on four functional groups: A,B,C and D that ESBLs is placed in A groups and derived from principal beta lactamases (TEM-1, TEM-2 and SHV-1) by substitution one or more amino acids in their catalytic cites. ${ }^{5}$ Among Enterobacteriaceae, Escherichia coli (E. coli) and Klebsiella spp. are more known to produce ESBLs. ${ }^{6} E$. coli is the most commonly encountered gram negative pathogen in nosocomial infections. ${ }^{7}$ Recently, the occurrence of ESBLs is rising worldwide; owing to identification of these strains in clinical laboratories to detect this resistance is very important. ${ }^{8}$ According to the recommendation of the Clinical and Laboratory Standards Institute (CLSI), ${ }^{9}$ one of the current methods for detection of ESBLs is an initial screening for the CLSI recommended cephalosporin's indicators especially ceftazidime and cefotaxime, subsequently performing of confirmatory tests with clavulanic acid (as one inhibitor of ESBLs). In this test, if zone of inhibition in the presence of clavulanic acid in compare absence clavulanic acid is increased, those organisms are recognized as a ESBLs producer. ${ }^{10,11}$ Complete detection of $\beta$-lactamase enzymes is essential for resistance control and successful treatment via the appropriate prescription of $\beta$-lactam drugs. Therefore, we focused this study to determine the incidence of ESBLs producing strains in clinical isolates.

\section{Materials and Methods}

\section{Bacterial strains}

Five hundred clinical samples were collected
Correspondence: Mohammad Mehdi Soltan Dallal, Dev. Microbiology, Department of Pathobiology, School of Public Health, Tehran University of Medical Science, Enghelab square, Tehran, Iran.

Tel. +98.21.6642268 - Fax: +98.21.66462267.

E-mail: soltanirad34@yahoo.com

Key words: Escherichia coli, $\beta$-Lactamase Enzymes, extended spectrum $\beta$-Lactamase.

Contributions: RB, microbiological test; JFM, molecular test results analysis; HMA, samples collection and primer design; AS, samples collection and molecular test; MMSD, upervising the project.

Conflict of interest: the authors report no conflicts of interest.

Received for publication: 26 March 2011.

Revision received: 28 May 2011.

Accepted for publication: 29 July 2011.

This work is licensed under a Creative Commons Attribution NonCommercial 3.0 License (CC BYNC 3.0).

CC Copyright R. Bakhtiari et al., 2011

Licensee PAGEPress, Italy

Microbiology Research 2011; 2:e8

doi:10.4081/mr.2011.e8

from Tehran hospitals (kid's medical center, Shariati, Mofid, Ali Asghar, Eghbal, Hakim, Valiasr, Khatamolanbia and Emam Khomeini) as of the following sources: urine, stool, blood, wound and other clinical samples of sick people. Two hundred $E$. coli isolates were detected by standard biochemical tests (IMVIC) by using TSI agar, SIM agar, Simmons Citrate agar, MR-VP medium and Urea media. Owing to store, all strain of $E$. coli isolated were cultured in skim milk and were stocked up at $70^{\circ} \mathrm{C}$ prior for testing.

\section{Antimicrobial susceptibility testing} for screening of extended spectrum beta lactamase

To performing initial screening of ESBLs was used from Disk diffusion method ${ }^{9}$ on $E$. coli isolates. In this method, after the preparation of Muller-Hinton agar plates, microbial suspensions corresponding with concentration of $0.5 \mathrm{McFarland}$ were completely distributed on the surface of plates. The following antibiotics and concentration were used: cefotaxime $(30 \mu \mathrm{g})$, ceftazidime $(30 \mu \mathrm{g})$, gentamicin (10 $\mu \mathrm{g})$, amoxicillin $(30 \mu \mathrm{g})$, imipenem $(10 \mu \mathrm{g})$, nalidixic acid $(30 \mu \mathrm{g})$, streptomycin $(10 \mu \mathrm{g})$, cotrimoxazole $(1.25 \mu \mathrm{g})$, ciprofloxacin $(5 \mu \mathrm{g})$ and chloramphenicol (30 $\mu g)$ (Mast Diagnostics Ltd., Bootle, Merseyside, UK). After $24 \mathrm{~h}$ incubation of plates at $37^{\circ} \mathrm{C}$, using a 
ruler, the zone of inhibition around each disk was measured and then compared with the standards of CLSI, followed by reported as resistant, intermediate or sensitive. ${ }^{9,12}$ A confirmation test (Combined Disk method) ${ }^{9}$ was performed on isolates of resistant to

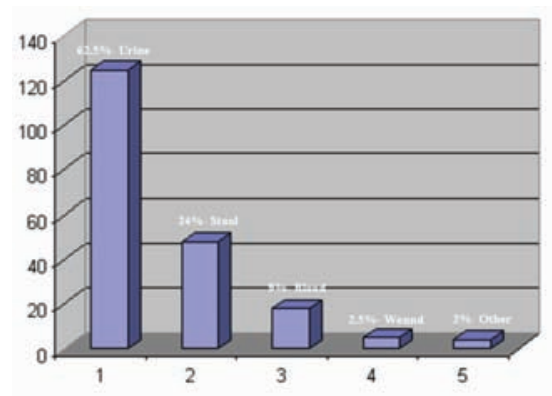

Figure 1. The frequency and source of $E$. coli isolates collected from Tehran hospitals during 6 months.

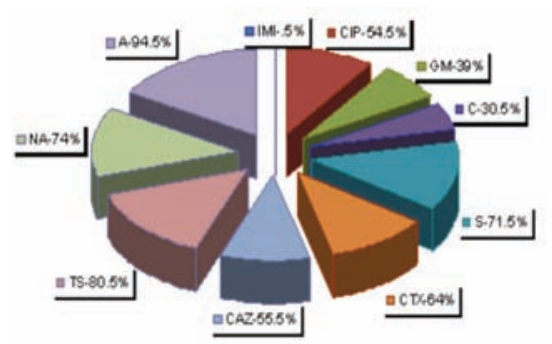

$=$ Imipenem

$\mathrm{CIP}=$ Ciprofloxacin

$\mathrm{GM}=$ Gentamicin

$\mathrm{C}=$ Chloramphenicol

$\mathrm{S}=$ Streptomycin

CTX $=$ Cefotaxime

$\mathrm{CAZ}=$ Ceftazidime

TS $=$ Cotrimoxazole

$\mathrm{NA}=$ Nalidixic acid

$\mathrm{A}=$ Amoxicillin

Figure 2. Pattern of resistance among 200 $E$. coli isolates to 10 antimicrobial agents.

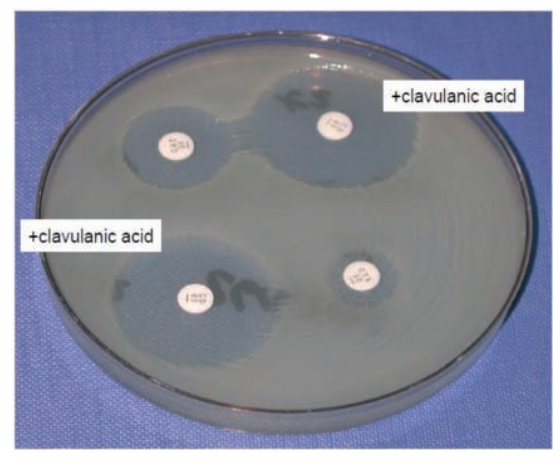

Figure 3. Combined Disk assay for detection of extended spectrum beta lactamase. CTX, Cefotaxime; CAZ, Ceftazidime; CV, clavulanic acid. cephalosporin's indicators which used from Ceftazidime $(30 \mu \mathrm{g})$, Ceftazidime/Clavulanate $(30 / 10 \mu g)$, Cefotaxime $(30 \mu g)$ and Cefotaxime/Clavulanate $(30 / 10 \mu g)$ (Mast Diagnostics Ltd.). After $24 \mathrm{~h}$ incubation of plates at $37^{\circ} \mathrm{C}$, ESBLs production was confirmed by $\geq 5 \mathrm{~mm}$ increase in the zone of inhibition in the presence of clavulanic acid compare to the absence of clavulanic acid. ${ }^{13,14}$

\section{Results}

Two hundred clinical isolates of $E$. coli were collected during a period of 6 months (Figure 1). Resistant pattern of $200 \mathrm{E}$. coli isolates to 10 antimicrobial agents are shown in Figure 2. Most of the isolates indicated high resistance to oxyiminocephalosporins, while they remained susceptible to imipenem. Up to $70 \%$ isolates exhibited the MDR (Multi Drug Resistance) phenotype.

In Disk diffusion method, 128(64\%) E. coli isolates which resistant to ceftazidime and cefotaxime were selected for possible positive ESBLs and followed by Combined Disk assay. In Combined Disk, among 128 screened isolates, 115(89.8\%) isolates were detected as ESBLs producers (Figure 3). $\beta$-lactamase-producing $E$. coli isolates was more common in urine samples (80\%).

\section{Discussion}

This study was conducted in Tehran hospitals such as kid's medical center, Shariati, Mofid, Ali Asghar, Eghbal, Hakim, Valiasr, Khatamolanbia and Emam Khomeini. Among 500 clinical samples from sick people, $200 E$. coli were isolates. ESBL screening and confirmation were performed by Disk diffusion method and Combined Disk assay, respectively. E. coli ESBLs positive culture cases were $89.8 \%$ alternatively. The resistant rate of $E$. coli to cephalosporin's indicators in this study higher than that $(32.11 \%)$ in the study of Nakhaei et al which reported from Iran. ${ }^{15}$ The results indicate that the ESBLs prevalence in community acquired infections particularly in E. coli is rising in Iran. In recent years, studies were reported that significant proportion of Enterobacteriacea isolates showed multidrug resistance against extended cephalosporins and aztreonam, that ESBLs production have the predominant role in this field. ${ }^{16}$ So, correct detection of them led to prevent from failure treatment and suitable prescription drugs.

The high prevalence of ESBL suggests that diagnostic laboratories should be better than phenotypic screen for ESBLs with ceftazidime as well as cefotaxime; ${ }^{17}$ they should still per- form clavulanate synergy tests on resistant isolates, but ESBLs may not be detected by standard laboratory methods because susceptibility results for cephalosporins may fall within the sensitive category and reported as false negative ESBLs which resulting from another factors such as AmpC that cover the effect of ESBL enzymes via creating resistance to clavulanate. ${ }^{16,18}$

In this survey among of 128 (64\%) screened E. coli isolates in Disk diffusion method, only 115 (89.8\%) isolate were selected for ESBLs producers by Combined Disk according to the CLSI. ${ }^{9}$ Negative ESBLs in other isolates (10.2\%) could be resulting other factors such as novel beta lactamase enzymes.

\section{Conclusions}

Today there are many $\beta$-lactam antibiotics and many more $\beta$-lactamase enzymes, including extended spectrum $\beta$-lactamases (ESBLs) and AmpC $\beta$-lactamases ${ }^{19}$ that make a many problem in detection of ESBLs. So, this investigation suggests using other methods including inhibitors of AmpC in confirmatory test for correct detection of ESBLs.

\section{References}

1. Li Q, Lee YJ, Castillo R, et al. NB2001, a novel antibacterial agent with broad-spectrum activity and enhanced potency against $\beta$-lactamase-producing strains. Antimicrob Agents Chemother 2002;46: 1262-8.

2. Perilli M, Celenza G, Santis DF, et al. E240V substitution increases catalytic efficiency toward ceftazidime in a New natural TEM-type extended-spectrum $\beta$-lactamase, TEM-149, from Enterobacter aerogenes and Serratia marcescens clinical isolates. Antimicrob Agents Chemother 2008;52:915-9.

3. Tenover FC, Raney PM, Williams PP, et al. Evaluation of the NCCLS extended-spectrum $\beta$-lactamase confirmation methods for Escherichia coli with isolates collected during project ICARE. J Clin Microbiol 2003;41:3142-6.

4. Bush K, Jacoby GA, Medeiros AA. A functional classification scheme for $\beta$-lactamases and its correlation with molecular structure. Antimicrob Agents Chemother 1995;39:1211-33.

5. Datta N, Kontomichalou P. Penicillinase synthesis controlled by infectious $\mathrm{R}$ factors in Enterobacteriaceae. Nature 1965;208: 239-41.

6. Eagye JK, Kuti LJ, Nicolau PD. Pharmaco- 
dynamic Comparison of the Carbapenems Against E. coli and Klebsiella spp. Containing Extended spectrum b-lactamases. Am J Infect Dis 2005;1:149-55.

7. Randrianirina F, Vaillant L, Ramarokoto $\mathrm{EC}$, et al. Antimicrobial resistance in pathogens causing nosocomial infections in surgery and intensive care wards in Antananarivo, Madagascar. J Infect Dev Ctries 2010;4:74-82.

8. Suvorov M, Vakulenko SB, Mobashery S. Cytoplasmic-membrane anchoring of a class A beta-lactamase and its capacity in manifesting antibiotic resistance. Antimicrob Agents Chemother 2007;51:2937-42.

9. Clinical and Laboratory Standards Institute (CLSI). Performance standards for antimicrobial susceptibility testing: Fifteenth Informational Supplement. 2005. M100-S15 Vol. 25 No. 1.

10. Stürenburg E, Mack D. Extended-spectrum beta-lactamases: implications for the clinical microbiology laboratory, therapy, and infection control. J Infect 2003;47:273-95.

11. Pitout JDD, Laupland KB. Extended-spec- trum $\beta$-lactamase-producing Enterobacteriaceae: an emerging public-health concern. Lancet Infect Dis 2008;8:159-66.

12. Netzel TC, Jindani I, Hanson N, et al. The AmpC inhibitor, Syn2190, can be used to reveal extended-spectrum $\beta$-lactamases in Escherichia coli. Diagn Microbiol Infect Dis 2007;58:345-8.

13. Song W, Bae K, Lee YN, et al. Detection of extended-spectrum $\beta$-lactamases by using Boronic acid as an AmpC $\beta$-lactamase inhibitor in clinical isolates of Klebsiella spp. and Escherichia coli. J Clin Microbiol 2007;45:1180-4.

14. Deshpande LM, Jones RN, Fritsche TR, Sader HS. Occurrence of plasmidic AmpC type $\beta$-lactamase-mediated resistance in Escherichia coli: report from the SENTRY Antimicrobial Surveillance Program (North America, 2004). Int J Antimicrob Agents 2006;28:578-81.

15. Nakhaei MM, Moshrefi SH. Determine the pattern of antibiotic resistance urine $\mathrm{E}$. coli isolates and survey the prevalence of broad spectrum beta lactamases. J Med Centers Sabzevar 2009;4:228-33.
16. Hawser PS, Bouchillon KS, Hoban JD, et al. Emergence of High Levels of ExtendedSpectrum $\beta$-Lactamase-Producing GramNegative Bacilli in the Asia-Pacific Region: data from the study for monitoring antimicrobial resistance trends (SMART) Program, 2007. Antimicrob Agents Chemother 2009;53:3280-4.

17. Rawat D, Nair D. Extended-spectrum $\beta$ lactamases in Gram Negative Bacteria. J Glob Infect Dis 2010;2:263-74.

18. Liu G, Ling BD, Zeng Y, et al. Mulcular characterization of Extended -Spectrum lactamases Produced by clinical isolates of Enterobacter cloacae from a Teaching Hospital in china. Jpn Infect Dis 2008;61: 286-9.

19. Jeong SH, Song W, Kim JS, et al. Broth Microdilution Method To Detect ExtendedSpectrum -Lactamases and AmpC Lactamases in Enterobacteriaceae Isolates by Use of Clavulanic Acid and Boronic Acid as Inhibitors. J Clin Microbiol 2009;47: 3409-12. 